\title{
Germanica
}

\section{Sujet et polyphonie dans Ariane à Naxos et La femme sans ombre D'Hofmannsthal*}

Subjekt und Polyphonie in Ariadne in Naxos und Frau ohne Schatten

\section{Christiane Chauviré}

\section{OpenEdition}

\section{Journals}

Édition électronique

URL : http://journals.openedition.org/germanica/1964

DOI : 10.4000/germanica. 1964

ISSN : 2107-0784

Éditeur

Université de Lille

\section{Édition imprimée}

Date de publication : 30 juin 1996

Pagination : 13-36

ISBN : 9782098426320

ISSN : 0984-2632

Référence électronique

Christiane Chauviré, «Sujet et polyphonie dans Ariane à Naxos et La femme sans ombre

D'Hofmannsthal* », Germanica [En ligne], 18| 1996, mis en ligne le 03 juin 2013, consulté le 06 octobre 2020. URL : http://journals.openedition.org/germanica/1964; DOI : https://doi.org/10.4000/ germanica.1964

Ce document a été généré automatiquement le 6 octobre 2020.

(c) Tous droits réservés 


\title{
Sujet et polyphonie dans Ariane à Naxos et La femme sans ombre D’Hofmannsthal*
}

\author{
Subjekt und Polyphonie in Ariadne in Naxos und Frau ohne Schatten
}

\author{
Christiane Chauviré
}

\section{NOTE DE L'AUTEUR}

* Je remercie Monsieur Michel Valensi et les Éditions de l'Éclat qui ont aimablement autorisé la reproduction de fragments de mon ouvrage Hofmannsthal et la métamorphose (L'Éclat, 1992).

1 La « mort du sujet » est couramment attribuée au mouvement structuraliste des années 1960. Mais pour qui a lu les Viennois du début du siècle cette pensée audacieuse est des plus familières. Dès avant 1900 le physicien-philosophe Mach avait dissout le moi en une multitude d'impressions évanescentes. Plus tard Musil chercha dans le roman essai la forme littéraire propre à exprimer la dilution du sujet dans un «monde de qualités » corrélatif de l'«homme sans qualités ». À Vienne, la destruction du sujet par Mach allait dans le même sens que toute une littérature inspirée de Taine et de Ribot sur le moi dissocié, dispersé, dédoublé dont La Lettre à Lord Chandos d'Hofmannsthal est l'exemple emblématique. En plus de la vulgate clinico-psychiatrique de l'époque, et de l'impressionnisme de Mach, Hofmannsthal y recueillait l'influence de la Sprachkrisis finde-siècle et $d u$ thème baroque, shakespearien de l'instabilité du moi. Pour Lord Chandos, toute frontière disparaissait entre le moi et les choses dans la plus totale perte de contrôle du langage.

2 Le théâtre, et surtout l'opéra et sa "polyphonie ", offraient à Hofmannsthal d'autres moyens d'expression à cette crise du moi. Déjà les récits de psychiatres sur les femmes somnambules ou hypnotisées, les présentations publiques de malades mentaux mettaient en scène des personnalités très "théâtrales" au moi incertain. 
Hofmannsthal exploita au théâtre et dans ses livrets d'Opéra - comme dans ses contes et ses nouvelles - le thème des moi multiples et fragmentés. L'espace scénique lui permettait de démultiplier en personnages contraires et complémentaires le moi divisé d'une seule hérö̈ne (Ariane et Zerbinetta dans Ariane à Naxos qui illustre le thème de l'unité du moi perdue puis retrouvée, ou de la recomposition d'un sujet après dispersion centrifuge. La femme sans ombre offre une autre variation sur le même thème : un moi incomplet, dont le manque est symbolisé par l'absence d'ombre, aspire à la complétude. Le travail du matériau sonore, l'utilisation par Richard Strauss de voix de femmes graves et aiguës accusent les contrastes ou opèrent les fusions là où les mots sans la musique ne suffiraient peut-être pas.

\section{Ariane ou le moi clivé}

3 S'il est un problème métaphysique qui a hanté Hofmannsthal, nourri de la jeune psychanalyse, c'est celui de la perte d'unité du moi, qui dans son œuvre revêt la forme du dédoublement de personnalité, de la Spaltung. Employant le mot de Freud et des psychiatres de l'époque - Spaltung - Hofmannsthal évoque « un excès dans le discours, une exagération: "dans cet excès, il y a un clivage (Spaltung), une partie du moi commet ce que l'autre ne veut pas » (Ad me ipsum, Reden, III, p. 615). Ce « thème Dr Jekyll et Mr Hyde ", comme il l'appelle (Reden, III, p. 468), alimenté par la lecture d'ouvrages sur les maladies de la personnalité (Ribot, Janet, Morton Prince, Breuer, Freud), est central dans deux de ses nouvelles, Andreas (roman inachevé de 1912, presque contemporain d'Ariane, dont le sous-titre, Die Vereinigten, est déjà significatif, et où l'on retrouve la même bipolarité que dans Ariane avec Maria et Mariquita) et Lucidor (dont Hofmannsthal tirera par la suite le livret d'Arabella). La célèbre Lettre à Lord Chandos (1903), qui traite de la dislocation du moi, envahi par les objets extérieurs et distendu aux limites du monde, en est la première expression littéraire. Le problème du moi dissocié, sans unité interne, et dilaté aux limites de l'univers (Das ich als Universum) s'est en effet imposé à Hofmannsthal après qu'il eut suivi les cours du philosophe « impressionniste » Ernst Mach, dont la formule : «le moi est insauvable » connut une certaine vogue dans les milieux littéraires de la Jung Wien. De la Lettre à Lord Chandos, qui illustre par une fiction les thèses dévastatrices de Mach sur l'absence d'unité et d'identité du moi, mais aussi «le doute contemporain jeté sur le langage » (Reden, III, p. 510), on trouve plusieurs équivalents dans la littérature de l'époque, chez Schnitzler (Anatol), Beer-Hofmann, Zweig, Svevo et Musil (les deux premiers étant des amis personnels de Hofmannsthal). "Le moi peut être étendu d'une manière telle qu'il englobe finalement l'univers entier ", écrit Mach dans son Analyse der Empfindungen, «Le moi n'est pas délimité de façon nette, la frontière est assez indéterminée et peutêtre déplacée à volonté », poursuit-il. C'est sur cette impossibilité, remarque Jacques Bouveresse, qu'est construit l'Homme sans qualités de Musil. Et Wittgenstein à son tour reprendra le problème sur le mode philosophique dans son Tractatus logico-philosophicus de 1912.

4 On notera la parenté entre l'impressionnisme de Mach et certains thèmes de la littérature baroque chère à Hofmannsthal :

Quand je dis que le moi est insauvable - écrit Mach à Hermann Bahr en 1908 - je veux dire par là qu'il réside dans la perception pour l'homme de toutes les choses, de toutes les manifestations, que ce moi se dissout dans tout ce qu'on peut ressentir, voir ou toucher. Tout est éphémère. Un monde sans substance qui n'est 
constitué que de couleurs, de contours et de sons. La réalité est en mouvement perpétuel, en reflets changeants à la manière d'un caméléon. C'est dans ce jeu de phénomènes que se cristallise ce que nous appelons notre « moi ». De l'instant de notre naissance à notre mort, il se transforme sans cesse.

5 Le vécu est une suite de tableaux impressionnistes, sinon pointillistes. Hofmannsthal, qui a retenu la leçon de Mach, conjugue les deux thèmes de l'Ichlosigkeit et de la Sprachlosigkeit - nous ne possédons ni notre moi ni notre langage - qu'il étend à l'analyse de son époque, caractérisée par deux mots: Auflösung (dissolution) et das Gleitende (le glissant).

La nature de notre époque est l'ambiguïté et l'indétermination. Elle ne peut s'appuyer que sur du glissant et elle a conscience que ce que les générations antérieures croyaient être force n'était en fait que du glissant.

écrit-il en 1905 dans Le Poète et son temps.

7 Le clivage est celui des aspects de la dissolution du moi auquel Hofmannsthal s'attache tout particulièrement. Sa nouvelle Lucidor présente un cas de double personnalité : Wladimir croit aimer une seule femme, Arabella, alors que la femme tendre et passionnée qu'il rencontre la nuit est en réalité Lucille, la sœur de la froide Arabella, qu'il ne voit que le jour. Cet homme tiraillé par deux exigences incompatibles, dont l'une est littéralement refoulée (« les pulsions de Wladimir nocturne se tapissaient dans l'ombre [...] toujours prêtes à plonger sous la surface obscure pour disparaître dans l'inconscient ", «Lucidor ", in Andreas et autres récits, tr. fr. Gallimard, p. 235), ne peut trouver son compte que dans l'alternance de la belle de jour et de la belle de nuit. Dans Lucidor, le schéma d'Ariane (deux femmes apparentes, une seule femme réelle) est en quelque sorte retourné (deux femmes réelles, une seule femme apparente).

Comme plus tard la Femme sans ombre, Ariane est construite sur un jeu d'oppositions structurales entre deux univers incompatibles (« réunis dans l'incompréhension») et deux femmes antithétiques : «Ma qualité de librettiste, écrit Hofmannsthal, n'est peutêtre pas si difficile à définir [...]. Je bâtis sur des contrastes pour découvrir sur ces contrastes l'harmonie du tout» (15 juin 1911). L'opposition entre "le tendre univers lyrique et fantasmatique d'Ariane " et "l'univers mélodique et transparent» de Zerbinette se traduit dans la musique par le contraste entre l'harmonium qui caractérise Ariane et le piano (instrument de Zerbinette). Mais ce dualisme n'est qu'apparent : il n'y a pas là deux mondes, mais un seul monde dédoublé. Ce doppelte Welt (Ad me ipsum, p. 614), dont l'un est le reflet (Spiegelbild, ibid., p. 607) - en un sens quasi platonicien - de l'autre, Hofmannsthal l'évoque à propos de la mythologie :

Par exemple, les dieux chez Homère : seulement le reflet des héros; de même, dans les religions, les reflets anthropomorphes, d'innombrables manières. Double monde qui en résulte, et qui seul est aimable, puisque c'est également l'amour qui produit un tel reflet. Et les Nibelungen, si terribles, parce que c'est un poème sans reflet, et les héros, comme des êtres de bronze qui n'existent que par et pour eux-mêmes. (Livre des amis, tr. fr., p. 88 ; cf. aussi Ad me ipsum, Reden, III, p. 614).

Si Hofmannsthal et Strauss accusent les contrastes, par toutes sortes de procédés, c'est pour suggérer par ailleurs l'unité des contraires, l'identité des deux univers, des deux couples, des deux femmes et de leurs deux histoires, la même histoire contée à des niveaux de compréhension différents. Mêlant au néoplatonisme le thème baroque du masque ( «ici tout n'est que masque de la vie d'en haut comme de celle d'en bas ») et celui, romantique, du Double (Doppelgänger), Hofmannsthal présente dans ses notes et 
dans l'Ariadnebrief le monde des Italiens comme le reflet ou le double vulgaire du monde idéal d'Ariane.

Le thème de l'unité profonde d'êtres en apparence dissemblables - dont on peut voir l'origine dans la question nietzschéenne de l'individuation et de l'aspiration à retrouver l'unité primordiale - trouve dans Ariane, mieux encore que dans Elektra, une expression dramatique privilégiée. Tout l'art d'Hofmannsthal est ici de suggérer, sans les affirmer didactiquement, les « correspondances ", au sens baudelairien, entre ces deux mondes, de faire apparaitre en filigrane les liens invisibles qui les rattachent. L'identité, enfin, de la noble et fidèle Ariane et de l'immorale Zerbinette tente de s'identifier à Ariane : lorsque, dans le Prologue, elle apparait au Compositeur charmé comme une autre Ariane ( Tu es comme moi, rien de terrestre n'existe en ton âme » lui dit-il, et lorsque, dans son aria, elle souligne à l'intention d'Ariane la parenté de leurs deux histoires (« Il est beaucoup d'îles désertes de ce genre, même parmi les humains, moi-même j'en ai habité plusieurs »). Séparés en apparence par les époques, les genres, les conditions sociales, Ariane et Zerbinette sont unies en profondeur : il s'agit d'une seule et même femme, au double visage, à la double vie. Sans doute l'intransigeante Ariane refuse-telle de se reconnaître dans le miroir que lui tend Zerbinette, repoussant, comme déjà le Compositeur dans le Prologue, la version triviale de son histoire, cette image déformée d'elle-même que lui renvoie Zerbinette. Aucune osmose ne s'opère entre leurs deux univers, parce que l'univers d'Ariane n'est pas perméable, parce qu'elle refuse l'identification suggérée par Zerbinette : c'est pourquoi il n'y a pas de mélange des genres et d'ailleurs on reste définitivement dans l'opera seria dès l'arrivée de Bacchus (si l'on excepte l'intervention finale, comme gommée, de Zerbinette).

11 Or du point de vue de Hofmannsthal, c'est paradoxalement Zerbinette qui a raison, et qui, dans son désir de s'identifier à Ariane, porte le message de Hofmannsthal : c'est elle qui affirme l'identité, tandis qu'Ariane affirme la différence. C'est Zerbinette qui insinue qu'elle et Ariane sont la même femme, tout comme Elektra et Chrysothémis, en dépit de leur affrontement, sont une femme intérieurement divisée qui se diffracte sur la scène :

Là où des contrastes se sont présentés [...] comme avec Elektra, l'héroïque, et Chrysothémis, la féminine [...], il s'agissait toujours pour moi de penser qu'ensemble ils formaient une unité, qu'ils étaient réellement un. (Ad me ipsum, 1922, p. 618).

Le « thème de l'unité des contraires, le sentiment de l'harmonie des contraires » (Reden, III, p. 509) omniprésents chez Hofmannsthal, reflète une vision mystique des polarités que ses lectures sur l'alchimie lui ont sans doute inspirée :

\footnotetext{
Adolescent, je voyais l'unité du monde. Le Religieux dans sa beauté, la beauté innombrablement diverse de tous les êtres me poignait, aussi leurs contradictions intérieures, et ceci que tous étaient cependant en connexion, en rapports profonds les uns avec les autres. Plus tard ce fut l'individuel et les formes agissant à l'arrièreplan de cette merveilleuse unité, que je me sentais contraint de révéler, de représenter. Cependant, je ne perdais pas de vue cette unité (Ad me ipsum, 1922, p. 618).

La seule identité qui résiste à l'acuité d'un regard plus profond est l'identité des contraires (Livre des amis, tr. fr., p. 40).
}

Par l'unité des contraires une sorte de compensation s'opère dans l'univers: tout est alors en équilibre - il s'agit bien là d'une pensée alchimique. Le mystère du monde, que Hofmannsthal ressent si fort, réside peut-être dans la réunion de ce qu'on ne peut 
réunir : « la vie est sans repos », elle est «Vereinigung des Unvereinbaren» (Reden, III, 1928, p. 592).

Plutôt qu'une réminiscence de la contradiction hégélienne, de l'idée que « toute chose est en elle-même contradictoire ", et qu'une synthèse dialectique fondra ensemble thèse et antithèse, c'est peut-être, chez Hofmannsthal, outre un emprunt à l'alchimie, une concession à la psychanalyse naissante et à son concept d'ambivalence : à propos du mythe, Hofmannsthal, lecteur de Freud, écrit : «Dans le domaine mythique, chaque chose est portée par un double sens qui est son sens opposé : vie = mort, combat de serpents = enlacement amoureux. C'est pourquoi dans le domaine mythique tout est en équilibre " (Livre des Amis, tr. fr. p. 38). Hofmannsthal est ici plus proche de Dürkheim dans son analyse du mythe ("Il est sans cesse question d'êtres qui ont les attributs les plus contradictoires, qui sont à la fois un et plusieurs, matériels et spirituels...») dans Les formes élémentaires de la vie religieuse, que de Hegel lorsqu'il compare la vérité au " délire bacchique dont il n'y a aucun membre qui ne soit ivre » et qui pourtant est aussi bien "repos translucide et simple ». Hofmannsthal ne s'intéresse pas à ce qui, comme chez Hegel, devient le contraire de soi au cours d'un processus dialectique. Et s'il est depuis toujours fasciné par le délire bacchique, occulté dans Ariane, et par la terrible aliénation que l'homme connaît dans une telle extase (" cet état de l'homme est au sens propre hors de lui ", note Hofmannsthal), c'est d'abord à cause de la menace qu'il fait peser sur l'unité du moi : «la croyance en l'indissolubilité et à la fixité de l'individu s'efface. Le sol vacille sous nos pieds ».

\section{Femme sans ombre, femme sans qualités}

15 La prédilection de Hofmannsthal pour le théâtre baroque - qui culminera plus tard en 1927 dans son chef-d'œuvre La Tour, et déjà visible dans La Mort et le Fou - est nettement perceptible dans La Femme sans ombre. Et c'est à juste titre que P.-Y. Pétillon rapproche Hofmannsthal du baroque élisabéthain (il va de soi que l'ombre de Shakespeare plane sur le théâtre de Hofmannsthal, ainsi que celle de Calderon). L'auteur aussi multidoué qu'était Hofmannsthal, qui s'était essayé à de multiples genres théâtraux (tragédie grecque : Elektra, « jeu » médiéval, livret d'opéra, drame baroque) revient toujours au baroque. Dans $L a$ Tour, Basile, le roi-père (comment ne pas lui prêter le visage de François-Joseph...), cherche à briser son fils Sigismond, (réminiscence de Hamlet); dans La Femme sans ombre, un autre roi-père, Keikobad, éprouve trop durement sa fille, l'Impératrice, lui accordant toutefois in extremis la liberté de se racheter et de racheter tous les autres personnages. Le martyre de celle-ci a donc bien un sens : il a la valeur d'une rédemption. C'est pourquoi du point de vue dramatique La Femme sans ombre se rattache aux Passions jouées à Pâques dans les villages du Tyrol ou de Bavière, encensées par Hofmannsthal comme des formes authentiques et naïves du théâtre populaire et qui relèvent de la même veine que les mystères médiévaux (Hofmannsthal en a justement écrit un: Jedermann). Dans ces Passions, le sacrifice comme acte cérémoniel occupe une place centrale. Et La Femme sans ombre est sans doute plus proche de ces Passions catholiques - dont elle présente, certes, une version très sophistiquée - que La Flûte enchantée, marquée par le rituel maçonnique des épreuves et par l'idéologie de l'Aufklärung (hostile à l'obscurantisme catholique).

En faisant de l'Impératrice une figure christique, un agneau sacrificiel, Hofmannsthal a voulu aussi symboliser ce qu'est pour lui le destin du poète: la descente de 
l'Impératrice au milieu des humains, sa décision de partager leur sort retrace symboliquement la démarche du poète, médiateur dont l'humanité a besoin, et qui prend sur lui toutes les souffrances de l'époque, vibrant, tel un sismographe - écrit Hofmannsthal - à tous les événements du monde : « car il souffre de toutes les choses et souffrant d'elles il en jouit. Cette jouissance dans la souffrance, voilà le contenu de sa vie ", c'est là tout le destin de l'Impératrice dans La Femme sans ombre. Comme le poète, elle a le devoir de quitter son royaume protégé - sa tour d'ivoire - son isolement, pour s'exposer aux malheurs du monde. La présence de souverains, et qui plus est d'un Empereur, n'est ni hasard ni respect de la convention du conte oriental ou de la comédie à la Gozzi. Le privilège de la naissance est signe d'élection (c'est déjà le cas de Tamino dans $\mathrm{La}$ Flûte enchantée) ; or, être élu, c'est être isolé, comme l'est le souverain dans la solitude absolue de son pouvoir, comme le fut François-Joseph, veuf inconsolable depuis des décennies et sans héritier. Le thème de l'isolement, si important dans La Femme sans ombre - l'Impératrice dans son île, l'Empereur coupé du monde - culminera dans La Tour avec Sigismond, le prince enfermé depuis sa naissance dans une tour, qui n'a rien vu du monde, ne connaissant que les mots et les vignettes du dictionnaire. Mais s'il y a le bon isolement signe d'élection, possession de qualités supérieures, il y a le mauvais isolement, la tour d'ivoire du poète (que Hofmannsthal condamne après l'avoir pratiquée), la solitude aristocratique de l'intellectuel ou de l'artiste coupé des autres, comme l'Empereur dont l'isolement volontaire traduit le mépris des hommes. Le motif impérial prend d'ailleurs toute sa valeur si on le replace dans le contexte viennois de la double monarchie. Hermann Broch a évoqué la fascination du jeune Hofmannsthal pour la figure, légendaire de son vivant, du vieil empereur solitaire qui symbolise la grandeur et la gloire du passé de l'Autriche, totalement identifié à son rôle d'empereur.

Hofmannsthal a d'abord hésité entre « un opéra fantastique et comique dans le style de Gozzi », une œuvre fantastique à la manière de La Flûte enchantée ou à celle du Freischütz. En définitive il opte pour une forme théâtrale à vrai dire peu autrichienne, celle du Trauer spiel, l'ancien drame baroque allemand. La passion du baroque l'a emporté sur le sentiment autrichien et sur la volonté de produire un théâtre (artificiellement) populaire. Hofmannsthal, qui s'est en quelque sorte trahi lui-même, a beau jeu de reprocher après coup à Richard Strauss le grandiose de sa musique alors que lui, Hofmannsthal, imaginait comme pour Ariane une musique délicate, aérienne et cristalline, évoquant le théâtre de marionnettes autrichien ou la "ménagerie de verre ». Certes Strauss, qui wagnérise à outrance dans La Femme sans ombre, est à l'opposé des arts et traditions populaires d'Autriche, et aussi loin que possible de La Flûte enchantée ou de la pièce pour harmonica de verre de Mozart. Hofmannsthal aurait pourtant dû porter à son crédit sa mise en musique de la voix de cristal de l'Impératrice, réalisant sur des notes haut perché un chant désincarné sans respiration, à l'opposé du soprano corsé et sensuel de la femme de Barak, mais bien loin des acrobaties vocales de Reine de la nuit.

Ainsi est-ce à peine si l'on peut recevoir la volonté de Hofmannsthal de démarquer le Volksgeist autrichien du génie allemand. Sans doute a-t-il repris et intégré des éléments symboliques empruntés à la Flûte, mais l'intellectuel et l'aristocrate fin-de-siècle qu'était Hofmannsthal pouvait-il écrire un livret instinctivement populaire ? Et n'est-ce pas le signe d'une certaine faillite que de s'être essayé à plusieurs genres théâtraux, 
sans s'être fixé sur aucun, la seule constance étant le retour au baroque sous ses diverses formes?

Mais La Femme sans ombre n'a pas que des racines autrichiennes. Sa genèse est extrêmement complexe, Hofmannsthal ayant mêlé des sources très diverses : outre le Singspiel, la féerie viennoise, la Passion villageoise, le Märchen des romantiques allemands, les contes des Mille et une nuits, les comédies de Gozzi, la Commedia dell'Arte, le théâtre symboliste. La source la plus directe est Anna, un poème de l'Autrichien Lenau où la perte de l'ombre est explicitement associée au refus de la maternité par une femme qui veut rester belle.

Mais il convient de citer au premier chef le second Faust dont le souvenir hante à l'évidence maints passages de La Femme sans ombre, l'œuvre la plus goethéenne de Hofmannsthal. Une des plus ambitieuses aussi, puisqu'il ne s'agit de rien de moins que de ressusciter deux chefs d'œuvre aussi incontournables que La Flûte enchantée et Faust. Sans parler du Conte que Goethe a tiré de La Flûte enchantée: Hofmannsthal disait d'ailleurs de lui à peu près ce que Hermann Broch écrit de La Femme sans ombre: « un conte de fées d'artistes qui n'a ses racines dans aucun mythe populaire » (op.cit.). Certes le livret de La Femme sans ombre est plus sobre que le conte, écrit dans une langue somptueuse, mais artificiellement archaïque. La surcharge symbolique y est telle qu'elle impose plusieurs niveaux de lecture. Et il serait naïf de croire qu'elle puisse concerner vraiment un public populaire, même si la musique de Strauss vient au secours du livret qu'il élucide souvent de manière efficace. L'absence de tout élément comique a d'ailleurs dû peser à Strauss qui n'est pas parvenu à rééditer la réussite d'Elektra. Sa tendance à " wagnériser » contribue encore à éloigner La Femme sans ombre de son modèle mozartien ("Nous avons manqué de légèreté dans la touche », se plaint Hofmannsthal). Plus à l'aise dans l'intimisme et la gaieté, la féerie était moins familière à Strauss que l'univers petit-bourgeois du bonheur familial déjà exalté dans son poème autobiographique La Vie d'un héros. Assez paradoxalement, l'idéal Biedermeier revit dans la musique de La Femme sans ombre, avec ou sans la complicité de Hofmannsthal, accentuant ainsi les intentions moralisatrices du livret.

\section{La Femme sans ombre et La flûte enchantée : deux formes d'humanisme}

21 Si La Femme sans ombre s'inscrit bien en un sens dans la lignée des tentatives romantiques pour continuer la Flûte - le rêve de Goethe, qu'il ne put réaliser faute de compositeur - les deux opéras ne se ressemblent que superficiellement. Ils n'ont de commun que leur trame, ainsi qu'un matériel symbolique que Hofmannsthal a intentionnellement emprunté à la Flûte pour créer une atmosphère surnaturelle: enfants, palais, cabanes, prêtres, barques, torches, tunnels dans le roc, écrit-il à Strauss le 20 mars 1911. Leur contenu symbolique et leur enjeu idéologique s'avèrent très différents. Certes on peut isoler un schéma commun : dans un orient féerique, deux couples élus, de condition sociale différente, dont les destins sont liés, le destin du couple inférieur étant subordonné à celui du couple supérieur, sont soumis par des puissances occultes qui les manipulent, et dont la bienveillance n'est pas d'emblée évidente, à une série d'épreuves purificatrices qui doivent les initier, chez Mozart, au savoir et à la sagesse suprême, ainsi qu'à une forme sublimée de l'amour, chez Hofmannsthal, à ce qu'il nomme le mystère de la vie. Il s'agit dans La Femme sans ombre 
d'une initiation à l'humain et aux valeurs humaines qui, paradoxalement, élève les protagonistes tout en les humanisant. La différence d'enjeu idéologique est nette : à la dimension Aufklärung, très datée, de La Flûte enchantée, avec sa foi optimiste en la raison, sa soumission à un ordre cosmique et politique rationnel, s'oppose chez Hofmannsthal une forme laïcisée d'humanisme chrétien. À la marche ascensionnelle des deux couples de la Flûte, à l'arrachement aux ténèbres, à la conquête de la lumière, symbolisant l'accès à un niveau d'humanité supérieur qui rendra Tamino apte à gouverner en prince éclairé, Hofmannsthal substitue la descente du couple impérial dans le monde terrestre et le nécessaire apprentissage des valeurs humaines : fraternité, altruisme, sacrifice de soi. Apprentissage qui seul peut octroyer et garantir à quiconque la qualité d'être humain, si humble ou si puissant soit-il (c'est Barak qui sert de modèle humain à l'Impératrice: il me montre, dit-elle, ce que c'est qu'être homme). Parallèlement, condamnation du surhomme en la personne de l'Empereur qui incarne l'égoïsme aristocratique et le refus du contact avec les autres hommes. Pour Hofmannsthal, en effet, ce ne sont pas le savoir ou les lumières qui enseignent à devenir humain (en cela il s'écarte de l'Aufklärung et de son rationalisme triomphant), ce sont les épreuves et la souffrance morale surmontées, le dépassement de soi, le sacrifice qui forme ou éduque chacun des protagonistes et les métamorphose profondément. À cet égard La Femme sans ombre est beaucoup plus proche du Bildungsroman que La Flûte enchantée. Les épreuves imposées aux deux couples, qui sont le prix à payer pour cette humanisation, à la fois élévation et purification, sont foncièrement différentes des épreuves maçonniques codifiées, formelles, liées aux quatre éléments, car ce sont des épreuves intérieures qui ne poursuivent pas le même objectif. Le sujet n'est plus la marche des héros vers le savoir et vers l'amour sublimé, ni l'insertion de la femme dans un ordre défini par la raison masculine à laquelle elle doit se soumettre (quoiqu'à cet égard Hofmannsthal ne se montre pas très féministe!), il est bien plutôt la nécessité de s'humaniser en s'insérant dans la communauté humaine au lieu de rester dans sa tour d'ivoire: obligation, pour tout homme qui vit de la vie de l'esprit, artiste ou intellectuel, de dépasser cette vie pour se risquer à une existence engagée et solidaire des autres hommes, de participer au "mystère de la vie». L'esthète repenti qu'est Hofmannsthal dans les années 1910-1920, l'expartisan de l'art pour l'art, accomplit son auto-critique, justifie son engagement et sa politique artistiques, son évolution vers des formes d'expression plus sociales (notamment l'opéra) que la poésie. Quoique l'enjeu de La Femme sans ombre dépasse la simple dimension autobiographique, Hofmannshtal est bien "d'une certaine manière" l'Impératrice. Cet engagement, Hofmannsthal l'exprime à travers une parabole sur l'apprentissage de tous les liens moraux et affectifs qui soudent cette Gemeinschaft de la vieille Autriche dont les intellectuels viennois, Hofmannsthal, Musil, Zweig et d'autres ont la nostalgie. Les penseurs sociaux de cette époque l'opposent en effet à la Gesellschaft, société atomisée où les individus ne sont plus liés entre eux par des liens organiques.

Ainsi, s'éloignant de son modèle mozartien marqué par l'Aufklärung et l'idéal maçonnique, Hofmannsthal transmet, sous le couvert de la féerie, souvent utilisée d'ailleurs à des fins moralisatrices (on le voit dans L'Oiseau Bleu de Maeterlinck, de 1911), un message marqué par les valeurs, les cérémonies et les rituels catholiquesautrichiens, qui prennent la place des rituels maçonniques de la Flûte. Même si cette utilisation du catholicisme est au service d'un humanisme laïc. De la Flûte à La Femme sans ombre, il y a presque inversion des valeurs. Le sujet de ce second texte est la descente de l'Impératrice - être féerique, être de lumière - dans la nuit opaque de 
l'existence humaine : c'est le divin qui veut s'humaniser, l'esprit qui tend à s'incarner. Alors que dans le schéma ascensionnel de la Flûte, c'est l'humain qui tente de se spiritualiser, de se rapprocher d'un divin, il est vrai, impersonnel et rationnel, caché derrière Zarastro. Il y a donc là deux formes opposées d'humanisme. Autre différence capitale, le problème central de La Femme sans ombre, c'est le mystère de l'incarnation, l'acquisition du corps à travers celle de l'ombre qui symbolise la maternité : une femme sans enfants n'a pas de corps, n'est pas humaine. C'est l'aspiration faustienne, goethéenne, de l'esprit au corps et à la vie, en la personne de l'Impératrice, que Hofmannsthal place au centre du drame. La quête de l'ombre représente l'insatisfaction de l'esprit qui veut un corps. Quant à l'appartenance à la communauté humaine, c'est une des clés majeures de La Femme sans ombre. Wittgenstein notait à propos de Peter Schlemihl, le personnage de Chamisso qui vend son ombre au diable, que renoncer à l'ombre signifie renoncer à la communauté des hommes.

De même la quête de l'ombre signifie pour l'Impératrice l'insertion dans la communauté humaine et l'acceptation des valeurs dites humanistes. L'ombre qu'elle cherche n'est pas l'ombre qui suit partout Zarathoustra pour disparaître à midi et à minuit, heures de la transmutation chez Nietzsche; elle figure, comme l'a noté Otto Rank à propos des thèmes conjoints de la perte de l'âme, du reflet et de l'ombre dans la tradition des contes et récits merveilleux, la part obscure de nous-mêmes, le çà, l'inconscient, par opposition à l'âme. D'essence terrestre, l'ombre, collée à la terre, est dans La Femme sans ombre "ce rien noir", dies schwarze Nichts/hinter dir auf der Erde/... dies Ding ohne Namen... ["qui traîne derrière toi par terre/... cette chose sans nom... »] (la Nourrice).

Hofmannsthal s'insère donc dans une tradition littéraire et mythologique bien établie. Avec Chamisso et Hoffmann la perte de l'ombre est devenue un archétype du romantisme allemand avant d'être objet psychanalytique. L'analyse d'Otto Rank rapproche le fantasme de la perte de l'ombre du thème romantique du Double (cf. Hoffmann). Or le dédoublement de la personnalité est depuis toujours un thème qui fascine Hofmannsthal. Déjà illustré dans La lettre à Lord Chandos, Elektra, Ariane à Naxos, Andreas, Lucidor, et même dans ses pièces baroques comme La Mort et le fou, la division du moi, ou son incomplétude, trouve, avec l'absence d'ombre, une représentation nouvelle. La perte de l'ombre signifie la séparation d'avec soi (la femme de Barak), l'absence d'ombre, l'inachèvement du moi, l'absence d'identité propre (l'Impératrice). L'être complet a une ombre, c'est-à-dire un corps aussi indissolublement lié à l'âme que l'ombre peut l'être de chacun de nous. Part terrestre et part idéale ne peuvent exister l'une sans l'autre.

Un des ressorts de l'Opéra, repris dans La Femme sans ombre, est la juxtaposition d'un couple inférieur, vulgaire (le corps) et d'un couple supérieur, noble (l'âme), en fait dédoublement d'un seul couple (Mozart et ses librettistes ont exploité abondamment ce schéma). Dans la convention théâtrale (quelque peu bousculée dans Les Noces de Figaro: quel maitre vaudrait un valet comme Figaro ?) le couple inférieur (souvent un couple de valets) est la simple caricature du couple supérieur; dans La Femme sans ombre, c'est l'inverse qui a lieu, il sert de modèle d'humanité au couple impérial.

L'ombre est donc le corps, et, puisqu'il s'agit de femmes, le sein maternel, non pas le corps du désir amoureux (condamné chez l'Empereur), mais celui, transfiguré, de la perpétuation de la "Vie». L'ombre factice de la Teinturière symbolise sa stérilité, sanctionne chez elle la méconnaissance de la vocation de la femme, de la vraie nature 
du couple. Si la Teinturière perd à un moment son ombre, c'est dans l'inconscience de ce "pont jeté au-dessus de l'abîme du néant » (que chantent les veilleurs de nuit à la fin du premier acte) qui assure la continuité de la grande chaîne des êtres - vision baroque s'il en est - :

Ihr Gatten in den Häusern dieser Stadt, / liebet einander mehr als euer Leben/und wisset: Nicht um eures Lebens anvertraut, /sondern allein um eurer Liebe willen! (...) Ihr seid die Brücke, überm Abgrund ausgespannt, / auf der die Toten wiederum ins Leben gehen!

[Époux dans les maisons de cette ville, /aimez-vous plus que votre vie / et sachez: ce n'est pas au nom de votre vie/que le germe de la vie vous a été confié, / mais seulement au nom de votre amour! / (...) Vous êtes le pont dressé au-dessus de l'abîme / par où les morts reviennent à la vie !].

Cette suite ininterrompue des êtres et des générations, imagerie baroque, à l'opposé de la danse macabre médiévale, est la clé principale de La Femme sans ombre. Ce sont les veilleurs de nuit qui portent le message de Hofmannsthal.

\section{Le partage et l'échange}

Fréquente est l'existence dans les opéras d'une structure d'échange, mieux, d'une stratégie échangiste qui affecte les couples. Les livrets des opéras de Mozart en offrent les meilleurs exemples. Ce schéma est ici plus complexe. Dans Cosi fan tutte, on le sait, l'échange a lieu à l'intérieur de chaque couple, et en quelque sorte à égalité : chaque couple se défait, puis se refait; entre temps deux couples nouveaux se sont formés avec les mêmes protagonistes, qui sont plus vrais que les couples initiaux. À la fin, cette vérité se désagrège, les cartes sont redistribuées comme au début, la nouvelle donne reconduit l'ordre ancien, respectueux de la seule convention sociale. Les Noces, Don Giovanni, et même La Flûte enchantée comportent des échanges qui produisent temporairement (souvent à la faveur d'un déguisement, d'une mystification) des couples secondaires, dépareillés socialement : le Comte / Suzanne ; Figaro / la Comtesse déguisée en Suzanne; Leporello déguisé en Don Giovanni / Zerlina. Fausse égalisation que celle du travesti, destinée à disparaître dans l'irréductibilité des conditions sociales; les maîtres resteront maîtres et les valets valets. Dans La Femme sans ombre l'égalisation finale des destins des deux couples n'est pas qu'illusion fugace, c'est une véritable égalisation spirituelle (dans le maintien, il est vrai, des conditions sociales), et elle est définitive. Il n'y a d'ailleurs pas de croisement des deux couples (ébauché dans Ariane avec le flirt Arlequin-Ariane). Ce ne sont pas les couples qui s'échangent. La stratégie échangiste concerne ici l'enfant. Un des enfants à naître, c'est-à-dire l'ombre. Il y a vente d'ombre, pire : trafic d'ombres; et magie noire, la vente s'effectuant au moyen d'un pacte diabolique. Les pauvres vendent leurs enfants aux riches, se louent comme mères porteuses aux riches. Il y a là en jeu un grand fantasme culturel, non pas la seule vente ou location d'enfants, mais la substitution d'enfants à la naissance - un autre grand ressort du théâtre -. Les toutes premières ébauches de La Femme sans ombre (1911) évoquent d'ailleurs la vente d'un enfant déjà né, mieux, le jugement de Salomon, c'est-à-dire l'impossibilité du partage : une vraie mère ne peut couper son enfant en deux. La vraie mère est celle qui se dépasse elle-même en renonçant à son propre enfant: c'est là, en germe, tout le rôle de l'Impératrice au $\mathrm{III}^{\mathrm{e}}$ acte. La tragique exploitation des inégalités sociales ne retient pas Hofmannsthal, elle fait partie des données du problème; la Teinturière vend la seul chose qu'elle possède et qui ait une valeur : son ombre, car elle n'a pas les moyens de refuser de la vendre. La symétrie des 
deux couples, la réciprocité entre eux paraît donc bien hypocrite : d'entrée de jeu la balle est lancée dans le camp de la Teinturière, et il est clair que ne pas la renvoyer est au-dessus de ses moyens, à partir du moment où, piégée par la nourrice, elle découvre qu'elle possède quelque chose qui n'a pas de prix (alors que le Peter Schlemihl de Chamisso aurait pu se dispenser de vendre son ombre à l'homme en gris pour une poignée de pièces d'or dont il n'a pas réellement besoin). Et ce qu'on promet à cette femme frustrée: la jeunesse et la beauté éternelles, la richesse et l'amour pour toujours, n'ont pas non plus de prix (son contrat est théoriquement meilleur que celui de Faust...). En effet la nourrice d'entrée de jeu attise sa curiosité, évoquant Das Geheimnis des Kaufs/Und das Geheimnis des Preises/um den du dir alles erkaufst... [Le secret de l'achat et le secret du prix auquel tu pourras tout acheter...]. La Teinturière s'étonne d'abord que son ombre ait une telle valeur:

Der gekrümmte Schatten/eines Weibes, wie ich bin/Wer gäbe dafür/auch nur den schmählichsten Preis?

[L'ombre torse d'une femme comme moi, qui en donnerait même le prix le plus vil ?]

pour se vanter ensuite à Barak de la valeur de son ombre :

habe ich meiner Schatten verhandelt, /und es sind die Käufer willig/und der Kaufpreis ist herrlich/und ohnegleichen!

[J'ai mis mon ombre en vente, et il y a des acheteurs et le prix d'achat est splendide et sans égal !] fin de l'acte II).

La Teinturière veut tout, elle aura tout ; l'ombre, prétend la nourrice, lui permet de tout acheter: Alles, du Benedeite, alles zahlen begriege Käufer [Tout, ô bénie, les acheteurs avides paieront tout] ( $\mathrm{I}^{\mathrm{er}}$ acte).

31 Il s'agit pourtant d'un marché de dupes : l'ombre est de la fausse monnaie - ombre factice, non méritée - ; et ce que la Teinturière veut acheter, payer de cette monnaie, n'est que mirage (O Welt in der Welt / O Traum in Wacham [O monde dans le monde ! Rêve en pleine veille]) et fantasme, on échange une illusion contre une autre, ce que la nourrice lui fait miroiter n'est que "reflet dans un miroir»: Willst du um dies Spiegelbild/nicht den hohlen Schatten geben? [Ne veux-tu pas en échange de l'image qui est dans le miroir, donner ton ombre creuse ?] (voix de l'Impératrice). Une image sans valeur, contre une ombre sans valeur. Thèmes baroques par excellence, l'illusion, le mirage, le faux-semblant sont au cœur de la scène du IIe acte où la nourrice montre à la Teinturière l'apparence des richesses qu'elle aurait en vendant son ombre, imagerie baroque à peine gâtée par l'orientalisme kitsch de la musique de Strauss qui, en ce passage, rappelle tout à fait la danse des sept voiles de Salomé.

En revanche l'Impératrice va apprendre au III ${ }^{\mathrm{e}}$ acte à passer de la logique payer-le-prixde-l'ombre-en-fausse-monnaie, - celle de la nourrice, qu'elle a au début adoptée - à la logique du véritable prix pour l'ombre: le sacrifice de soi, l'auto-dépassement (Selbstüberwindung). Bref la logique du rachat; elle sait maintenant « de quel prix (les hommes) doivent tout payer pour se racheter d'une lourde faute ", et ne manque pas d'identifier ce rachat à une renaissance analogue à celle du phénix, forme suprême de la Verwandlung.

33 Ainsi la Teinturière s'engage-t-elle dans un marché de dupes en acceptant de vendre son ombre. Mais pire encore, elle se laisse impliquer par la nourrice, qui la manipule ("Mephisto femelle», écrit Hofmannsthal), dans une transaction dangereuse en concluant un pacte où le Diable est invoqué sans jamais être directement nommé : « Des 
puissances supérieures sont en jeu » annonce la nourrice, «On va invoquer des noms puissants, nouer une alliance, jeter un sort !» (acte I, même formule à l'acte II). Pacte aussi malhonnête que dangereux, qui choque d'ailleurs l'innocente Impératrice : « Les hommes sont-ils ainsi ? Avec un cœur si vénal?». De fait, ce n'est que parce que l'ombre de la Teinturière est factice, car imméritée (la femme ne veut pas d'enfants), que la transaction diabolique est possible. Il y a chez elle décalage entre le Zeichen (l'ombre) et le Bezeichnetes (la possibilité d'être mère ; cf. «Die Handlung», H.v.H. G. W., vol. V, p. 382). Car l'ombre authentique, elle, ne peut faire l'objet d'un trafic ou d'un marchandage. L'ombre malhonnêtement acquise, l'ombre fausse, est en réalité sans valeur. Keikobad, le roi des esprits, n'accepterait pour l'Impératrice ni une ombre factice ni une affaire dans laquelle le Diable a trempé. Ce que, du point de vue de Keikobad, l'Impératrice doit apprendre tout au long du drame, c'est que l'ombre ne s'achète pas, mais se mérite, et que le vrai prix à payer pour elle, c'est le sacrifice, l'auto-dépassement, au terme d'une série d'épreuves (sadiquement) organisées et mises en scène par Keikobad. Tous les protagonistes, d'ailleurs, doivent subir des épreuves (comme l'affirme le Messager de Keikobad) afin d'être métamorphosés, au sens le plus fort que peut avoir la Verwandlung chez Hofmannsthal: mort et résurrection. Précisément la Verwandlung prend ici la signification du rachat ou de la rédemption chrétienne. La rédemption finale des protagonistes s'accomplit grâce au sacrifice de l'Impératrice, qui, à la fin, renonce à l'ombre et offre sa vie pour sauver l'Empereur de la pétrification, rachetant ainsi tous les autres (conformément au dogme catholique, le sacrifice d'un seul suffit à les sauver tous). Mais il existe également dans La Femme sans ombre une Verwandlung réciproque ou, comme dit Hofmannsthal, "allomatique", interne à chaque couple, qui comporte dans les deux cas un être capable de racheter l'autre ou de la métamorphoser: Barak transforme sa femme comme l'Impératrice transforme l'Empereur. "La Femme sans ombre, triomphe de l'allomatique » écrit Hofmannsthal en 1916 dans Ad me ipsum :

Kaiser und Kaiserin im Schicksal (Fluch und Erlösung)

[L'Empereur et l'Impératrice dans le destin (Malédiction et rédemption)]

mit dem Sich-verwandeln das Verwandeln eines Andern

[Tout en métamorphosant, en métamorphoser un autre] (H.v.H. G. W., vol. III, pp.

607-608.

Die gegenseitige Verwandlung, das allomatische Element

[La métamorphose réciproque, l'élément allomatique].

Vereinigung und Verknüpfung sämtlicher Motive in der "Frau ohne Schatten» [Fusion et réunion, tout le motif de la «Femme sans Ombre»] (pp. 603-604).

Pris dans la machination derrière laquelle on devine l'invisible et tout-puissant Keikobad, qui ordonne et impose les épreuves, les destins des deux couples se trouvent étroitement noués. Mais la balance infernale qui lie le salut des uns à la perte des autres disparaît à la fin dans le salut et l'égalisation de tous :

Die Kaiserin und der Kaiser vereinigen in sich die Motive der Schicks alfindun g durch Aufsich-nehmen des Fluches zugleich das Gewahr-werden der Mitwelt als gleichberechtigt... [L'Impératrice et l'Empereur combinent en eux le motif du destin à trouver par le fait de prendre sur soi la malédiction et en même temps de s'apercevoir de ceux qui vous entourent comme égaux en droits] (p. 604).

Hofmannsthal présente lui-même ainsi le début du II ${ }^{\mathrm{e}}$ acte : Die Prüfungen gehen an; denn es müssen alle vier gereinigt werden, der Färber und sein Weib, der Kaiser und die Feentochter, $z u$ trübe wird sich das eine Paar, zu stolz und ferne der Erde das andere [Les épreuves commencent, car tous quatre ils doivent être purifiés, le Teinturier et sa femme, 
l'Empereur et la fille des fées, le premier couple si lourdement terrestre, l'autre si orgueilleux et lointain] («Die Handlung», H.v.H., G. W., vol. V, p. 383).

Ainsi, à un premier niveau, il est question dans La Femme sans ombre d'un trafic d'ombre entre la fille du roi des esprits, décidée à obtenir à tout prix une ombre-symbole de fécondité pour sauver l'Empereur, son époux, d'une malédiction qui le condamne à être pétrifié si elle ne devient pas mère dans un certain délai, et une femme pauvre, également sans enfants, mais qui n'en souhaite pas. Comme ce marchandage est malhonnête et diabolique, il s'effectue incomplètement, et les deux couples doivent parcourir un itinéraire initiatique jalonné d'épreuves dont le but est de les initier au «mystère » de la «Vie ». Au lieu de consister en exploits physiques courageux (La Flûte enchantée), ces épreuves se présentent comme des conflits intérieurs à surmonter, tel au IIP acte le combat de l'Impératrice tentée de boire la mystérieuse « eau dorée ». Boira, boira pas? Il s'agit pour elle de comprendre qu'elle ne doit pas boire (boire sauverait l'Empereur de la pétrification et donnerait une ombre à l'Impératrice mais ferait tomber le couple de la Teinturière sous le coup de la même malédiction: femme sans ombre plus mari pétrifié). L'épreuve oblige l'Impératrice à "se dépasser elle-même ", rachetant tous les autres. Au terme du parcours, elle décide d'appartenir au monde des humains : Mein Platz ist hier in dieser Welt! [Ma place est ici, dans ce monde !]. Le thème très antinietzschéen de la nécessaire humanisation se répercute de manière différente sur chacun des quatre protagonistes. L'Impératrice, pur esprit au départ, tient déjà de sa mère (humaine) «la nostalgie de l'humain" (d'où son mariage avec un homme, l'Empereur). Celui-ci, homme inhumain, vit solitaire dans l'ignorance ou le mépris des autres hommes, qu'il découvre à son grand étonnement en fin de $\mathrm{III}^{\mathrm{e}}$ acte, pour les reconnaître, dans un grand élan beethovenien, comme ses "frères ». Happy end : les quatre protagonistes ont été éprouvés et rachetés, tous ont accompli leur métamorphose, ont entendu la leçon de la "Vie », accepté la condition humaine, ce qui se traduit par la libération finale des enfants à naître. C'est la métamorphose finale des deux couples qui libère les enfants, dans cette ultime scène de retrouvailles où ceux qui s'étaient perdus et cherchés se retrouvent: chacun connaît, accomplit son destin : « maintenant ", dit l'Empereur à propos des enfants, « ils se précipitent dans la vie avec des ailes roses comme l'aurore, vers nous qui étions presque perdus ».

En effet c'est seulement si l'amour se sublime que des enfants peuvent naître. Ce que les enfants concrétisent, ce n'est pas, contrairement à l'évidence première, l'union physique, mais l'union spirituelle d'un homme et d'une femme. La stérilité est métaphysique, elle sanctionne un couple inaccompli, une union simplement érotique. Hofmannsthal disait avoir écrit La Femme sans ombre pour protester contre "l'esprit matérialiste de la médecine moderne ». La stérilité signale et punit l'égoïsme érotique. L'enfant est le reflet ou le témoignage d'une fusion mystique, comme le laisse entendre les veilleurs de nuit au premier acte. En outre la stérilité métaphysique n'est pas guérie par la magie de la nourrice, le trafic d'ombre. L'ombre, c'est-à-dire la maternité, doit se mériter. Au début la Teinturière a une ombre, mais pas d'enfant, l'Impératrice, ni ombre, ni enfant, toutes deux sont stériles, mais l'ombre de la Teinturière est comme de la fausse monnaie. La stérilité de la Teinturière est voulue et méritée, celle de l'Impératrice, involontaire, et non refus (c'est par la faute de l'Empereur si elle est stérile).

Cette variation poétique sur le thème de l'immaculée conception, ce rêve d'une conception en esprit, est une constante chez Hofmannsthal : «Poème en prose » écrit- 
il, « des amants qui ne s'appartiennent pas sont liés d'une attache métaphysique. Ils sont père et mère d'un enfant non né ". C'est pourquoi Hofmannsthal dramatise à l'extrême le refus a priori de la maternité, assimilé au meurtre actif d'un enfant à naître (c'est ce que les voix des poissons donnent à entendre à la Teinturière-mauvaise mère, criminelle en puissance).

Les enfants à naître sont l'enjeu et le moteur du drame. Présents et absents à la fois, ils dirigent les actes des protagonistes bien autant que Keikobad. Ils figurent l'appel de la Vie auquel chaque personnage finira par répondre. Les enfants se manifestent à trois reprises, et à chaque fois de façon décisive dans l'action. D'abord au premier acte sous l'apparence du faucon - il symbolise dans le Conte l'ainé des futurs enfants de l'Empereur - qui, en poussant celui-ci à s'engager dans une quête, est l'instigateur de sa métamorphose. Puis ce sont les voix des poissons qui au IIe acte troublent la Teinturière ; qu'elle puisse les entendre signifie qu'elle n'a pas réussi à faire taire en elle tout désir de maternité, ce qui prépare à sa métamorphose finale. Dans la scène ultime du III ${ }^{\mathrm{e}}$ acte, enfin, les enfants à naître, invisibles mais dont on entend les voix, se révèlent avoir joué le rôle de guides ou d'anges gardiens auprès de leurs futurs parents, veillant sur eux depuis le royaume des esprits où, comme dans L'Oiseau Bleu de Maeterlinck, ils attendent avec impatience d'être appelés à la vie. Investis d'une sagesse et d'une omniprésence célestes, ils savaient dès le début tout ce que les protagonistes ignoraient encore. Réminiscence évidente des trois enfants de La Flûte enchantée, ils symbolisaient l'innocence de l'état de préexistence. De par leur volonté d'exister, les enfants à naître constituent le principe actif du drame, la naissance étant pour eux délivrance; ils symbolisent, comme l'Impératrice, l'esprit qui tend à s'incarner, illustrent la supériorité de la Vie sur l'Esprit; cette Vie devant laquelle Keikobad luimême doit s'incliner, ou du moins avec laquelle il lui faut composer.

Par delà la quête du corps, les épreuves, le rachat, thèmes apparents de La Femme sans ombre, un sens plus profond se dégage de la quête de l'ombre: la quête d'un moi complet au prix de l'acquisition progressive de la condition humaine par l'Impératrice. Dans le dernier tableau, chacune des deux héroïnes a une ombre, ayant appris à la "mériter ", c'est-à-dire à réunifier un moi dissocié ou incertain. La crise du sujet n'a donc pas le dernier mot dans La Femme sans ombre. Elle ne trouvera son apothéose que dans le roman expérimental de Musil, qui dépasse le théâtre et l'opéra en capacité d'expression d'un moi fluctuant, polyphonique et peut-être définitivement annulé.

\section{RÉSUMÉS}

Dans les années 1960, la mort du sujet était une idée triomphante, marquant l'avènement d'un structuralisme source de renouvellement important en sciences humaines et en philosophie, Mais à Vienne, au tournant du siècle, la crise du sujet fut au contraire une pensée morbide conforme à l'obsession du déclin qui hantait cette génération. Elle se prêtait à toutes sortes de variations philosophiques et littéraires. La célèbre «Lettre à Lord Chandos » illustre dramatiquement la dissociation du moi, sa dilatation jusqu'aux limites du monde. Au théâtre, c'est par touches plus légères qu'Hofmannstahl met en scène des personnalités dissociées mises à 
la mode par la littérature psychiatrique de l'époque. Dans deux de ses livrets d'opéras écrits pour R. Strauss, Ariane à Naxos et La femme sans ombre, des femmes incarnent la crise du sujet avec l'aide supplémentaire de la polyphonie vocale.

In den 1960er Jahren feierte der Begriff des Todes des Subjekts wahre Triumphe. Er kam mit dem Strukturalismus auf, der einen wichtigen Beitrag zur Erneuerung der Humanwissenschaften und der Philosophie leistete. Im Wien der Jahrhundertwende aber wurzelte die Krise des Subjekts in einer morbiden Gedankenwelt, die den unter den meisten Köpfen dieser Generation spukenden Verfallsobsessionen entsprach. Der berühmte «Brief an Lord Chandos» zeigt in dramatischer Form die Spaltung des Ich bzw. dessen an die Grenzen der Welt stoßende Dehnung. Auf dem Theater inszeniert Hofmannstahl - diesmal mit leichteren Strichen - gespaltene Persönlichkeiten, wie sie durch die psychiatrische Literatur der Zeit in Mode gekommen waren. In seinen beiden Opernlibretti Ariadne auf Naxos und Frau ohne Schatten wird die Krise des Subjekts von Frauen verkörpert: die Polyphonie der Stimmen tut ein übriges dazu.

\section{AUTEUR}

\section{CHRISTIANE CHAUVIRÉ}

Université de Paris I 